The phenomenon of osmotic flow is therefore due to the residue or excess of solvent molecules on the pure solvent side; the solute molecules play an indirect part only. But the solute molecules do cause a strain to be placed on the membrane, which tends to rupture the latter.

The fundamental difference between osmotic phenomena in the gaseous and solution states is that whereas the active molecules (see diagram II.) have a vacuum for a medium in the gaseous case, they have a liquid solvent for a medium in the solution case. The other differences between compressed gases and concentrated solutions nearly all proceed from this fundamental one.

University of Birmingham, March $2 \mathrm{I}$.

\section{The Expansion of a Homogeneous Function in Spherical} Harmonics.

IN a recent paper, entitled "Notes on Spherical Harmonics" (Proceedings of the Edinburgh Mathematical Society, vol. xxxii., I9I4), Dr. John Dougall wrongly claims as new the expansion which he has given there for a homogeneous function of the coordinates of a point on a sphere. This expansion was first given in 1900 by Dr. G. Prasad, in the Messenger of Mathematics, vol. xxx., p. I3, and again, by a different method, in 1912 in the Mathematische Annalen, vol. Ixxii., p. 436 . The method of Dr. Prasad in the second paper is the same as that of Dr. Dougall.

Calcutta Mathematical Society,

University College of Science, Calcutta, March 8.

\section{PREVENTIVE EUGENICS. ${ }^{1}$}

I ORD SYDENHAM and his colleagues deL serve the thanks of the nation for their prompt and faithful discharge of the difficult task allotted to them, in November, 19I3, of inquiring into the prevalence of venereal diseases in the United Kingdom, their effects upon the health of the community, and the means by which those effects can be alleviated or prevented. No one can read the commissioners' report without an increased conviction of the seriousness of the evil that is dealt with, of its grave and farreaching effects (even on the biological plane alone) upon the individual and the race. In careful terms and with scientific precision the commissioners give the evidence for the statement that the effects of the diseases in question "cannot be too seriously regarded," for "they result in a heavy loss, not only of actual, but of potential population, of productive power and of expenditure actually entailed." The misery account cannot be estimated.

Except in the case of the Navy and Army, there are at present no means of arriving at an accurate estimate of the prevalence of venereal diseases in Britain, and many deaths due to them appear to escape official recognition. Sir William Osler considers that, "of the killing diseases, syphilis comes third or fourth," and the commissioners, while rightly cautious, conclude that the number of persons who have been infected

1 Royal Commission on Venereal Diseases. Final Report of the Commis sioners. Presented to both Houses of Parliament by Command of $\mathrm{Hi}$ Majesty. Pp. rgr. (London: Wyman and Sons, Ltd, rgr6.) Price is. ird. No. 2423, VOL. 97] with this disease, acquired or congenital, cannot fall below ro per cent. of the whole population in the large cities, and that the percentage affected with gonorrhœa must be much larger. As regards geographical distribution, syphilis is shown to be essentially a town disease. As regards the social distribution of venereal diseases as a whole, there is high incidence (in descending order) among unskilled labourers, in those intermediate between them and skilled labourers, and in the upper and middle classes. There is relatively low incidence among (in descending order) textile workers, miners, and agricultural labourers. It is regrettable that the statistics, both of total prevalence and of distribution, remain somewhat uncertain. It is also to be regretted that the commissioners have allowed themselves to speak repeatedly of "hereditary syphilis"-a quite inaccurate phrase.

One of the most tragic aspects of this widespread human scourge is the suffering inflicted on the innocent. Children infected before birth may be blinded or deafened, or terribly diseased in skin and bone, in body and mind. More than half of all cases of blindness among children are the result of venereal diseases in their parents. Of registered still-births, probably at least half are due to syphilis, and it is estimated that from 30 to 50 per cent. of sterility among women is due to gonorrhœa. The "suffering incalculable" that may be inflicted on an innocent mother, taken along with wrong done to the offspring and other possible consequences, have led the commissioners to the recommendation that the presence of communicable venereal disease should be regarded as a disqualification for marriage and as a ground for a declaration of nullity-without, of course, affecting the legitimacy of the children. Those who still think that nothing should be done to make the cure of the diseases easier, because this lessens the punishment of the guilty and makes indulgence safer, should consider carefully the section of the report which deals with the consequences to mothers and children. We confess that it overwhelms us in its awfulness. There is also to be borne in mind the terribleness of the nemesis involved in the occurrence in the offender himself of general paralysis or locomotor ataxy, it may be ten or fifteen years after the infection. An even wider consideration, especially in these days of wastage, is the "enormous" economic loss traceable to reduced working capacity, and the heavy public cost of maintaining the various kinds of patients. The commissioners are convinced that the cost of curative and preventive measures would soon be counterbalanced by what would be saved.

We are not here concerned with the medical measures by which, according to the commissioners, the diseases can be controlled and reduced within narrow limits, but we wish to direct attention to two accessory points:-(I) There is a wholesome eugenic breeze in the suggestion that a warning given by a physician in regard to the undesirability of a marriage shall be regarded as 